\title{
Discrete-time BSDEs with Random Terminal Horizon
}

\author{
Yin Lin, Hailiang Yang ${ }^{\dagger}$
}

\begin{abstract}
This paper studies the theory of discrete-time backward stochastic difference equations (also called BSDEs) with a random terminal time, which is not a stopping time. We follow Cohen and Elliott [2] and consider a reference filtration generated by a general discrete-time finite-state process. The martingale representation theorem for essentially bounded martingales under progressively enlarged filtration is established. Then we prove the existence and uniqueness theorem of BSDEs under enlarged filtration using some weak assumptions of the driver. We also present conditions for a comparison theorem. Applications to nonlinear expectations and optimal design of dynamic default risk are explored.
\end{abstract}

Keywords: BSDE, Progressively enlarged filtration, Random terminal horizon, Comparison theorem, Nonlinear expectations, Defaultable contingent claims, risk measures

\section{Introduction}

In this paper, we focus on theory of discrete-time finite-state BSDEs under progressively enlarged filtration. The motivation is an optimal design problem of dynamic risk measures of defaultable contingent claims. The application of BSDEs in such problem was first studied by Barrieu and El Karoui [1] in a Brownian setting. A related problem of optimal design of dynamic default risk measures using BSDEs on a single jump process was explored by Shen and Elliott [8]. In [8], the terminal payoff only depends on the occurrence of the default. However, in the real financial market, the payoff of the defaultable contingent claims may depend not only on the default time, but also on the paths of some risky assets. Therefore, in this paper, we use progressive enlargement of discrete-time finite-state filtration and characterize the optimal design of dynamic risk measures of such defaultable contingent claims using theory of BSDEs under enlarged filtration.

Sections of this paper are arranged as follows. We present assumptions on the financial risky assets, default time and enlarged filtration in Section 2. Martingale representation theorem under enlarged filtration is given in Section 3. Based on this theorem, we establish the existence and uniqueness theorem of BSDEs under enlarged filtration in Section 4. A comparison theorem is studied in Section 5. Applications of such BSDEs including nonlinear expectations and optimal design of dynamic risk measures are explored in Section 6. Finally Section 7 concludes this paper.

\section{Assumptions on reference market, default time and en- larged filtration}

We follow Cohen and Elliott [2] by considering a discrete-time finite-state process $X$ as the underlying stochastic process. Suppose $(\Omega, \mathcal{F}, \mathbb{P})$ is a probability space and $X=\left\{X_{t}, t \in\{0,1, \ldots, T\}\right\}$

\footnotetext{
*Department of Statistics and Actuarial Science, The University of Hong Kong, Pokfulam Road, Hong Kong, e-mail:linyin@connect.hku.hk

${ }^{\dagger}$ Department of Statistics and Actuarial Science, The University of Hong Kong, Pokfulam Road, Hong Kong, e-mail: hlyang@hku.hk
} 
is a finite state process. Without loss of generality we suppose for each $t \in\{0,1, \ldots, T\}$,

$$
X_{t} \in\left\{e_{1}, e_{2}, \ldots, e_{N}\right\},
$$

where $N$ is the number of the states and $e_{i}$ is the $i$ th standard unit vector in $\mathbb{R}^{N}$. Consider a filtered probability space $\left(\Omega, \mathcal{F},\left(\mathcal{F}_{t}\right)_{0 \leq t \leq T}, \mathbb{P}\right)$, where

$$
\mathcal{F}_{t}=\sigma\left(\left\{X_{s}, s \leq t\right\}, A \in \mathcal{F}: \mathbb{P}(A)=0\right) .
$$

Then, we can define the martingale process $M$ by $M_{t}:=X_{t}-\mathbb{E}\left(X_{t} \mid \mathcal{F}_{t-1}\right)$.

We consider a problem of risk measure of a defaultable contingent claim which is associated with a random default time $\tau$, which is not an $\mathcal{F}$-stopping time. The terminal payoff of the claim at time $\tau \wedge T$ has the following form:

$$
Q:=V I_{\tau>T}+C_{\tau} I_{\tau \leq T},
$$

where $V$ is $\mathcal{F}_{T}$-measurable, i.e. $V$ depends on the paths of asset prices until time $T$, and $C$ is an $\mathcal{F}$-predictable nonnegative process.

In this paper, we consider BSDEs with uncertain time horizon: the terminal time $\tau$. The default time does not depend entirely on the paths of the underlying financial assets. However, at each time $t$, it should be observable whether the default $\tau$ has occurred or not. Therefore, the information is not the filtration generated by the price processes $\left(\mathcal{F}_{t}\right)_{0 \leq t \leq T}$, but is defined as $\mathcal{G}=\left(\mathcal{G}_{t}\right)_{0 \leq t \leq T}$, where

$$
\mathcal{G}_{t}=\mathcal{F}_{t} \vee \sigma((t+1) \wedge \tau),
$$

which is the completion of the smallest filtration that contains filtration $\left(\mathcal{F}_{t}\right)_{0 \leq t \leq T}$ and that makes $\tau$ a stopping time.

In order to avoid arbitrage opportunities, we should work on a mathematical set up where $(\mathcal{F}, \mathbb{P})$ semi-martingales remain $(\mathcal{G}, \mathbb{P})$ semi-martingales. Initial time setting was adopted in continuoustime cases. Similarly, in this paper, we consider the following hypothesis about conditional probability function.

Hypothesis H1. Assume that for all $t$, there exists an $\mathcal{F}_{t} \times \mathcal{B}\left(\mathbb{R}^{+}\right)$-measurable function $\alpha_{t}$ : $(\omega, s) \rightarrow \alpha_{t}(\omega, s)$ which satisfies for all $s, \mathbb{P}$-a.s.

$$
\mathbb{P}\left(\tau=s \mid \mathcal{F}_{t}\right):=\alpha_{t}(s) .
$$

Remark 1. In the continuous-time setting in [6], density hypothesis guarantees that $\tau$ avoids $\mathcal{F}$-stopping times, which means $\tau$ does not share common jump with any $\mathcal{F}$-adapted process. However, in the discrete-time setting, Hypothesis $\mathrm{H} 1$ does not necessarily implies such property. In this paper, we adopt the following hypothesis.

Hypothesis H2. $\tau$ does not share common jump with $X$, that is,

$$
\mathbb{P}\left(X_{t+1} \neq X_{t}, \tau=t+1\right)=0, \forall t \in[0, T] .
$$

We define the conditional cumulative distribution function and conditional survival function:

$$
F_{t}:=\mathbb{P}\left(\tau \leq t \mid \mathcal{F}_{t}\right)=\sum_{s=0}^{t} \alpha_{t}(s), \quad S_{t}=1-F_{t}=\sum_{s=t+1}^{\infty} \alpha_{t}(s) .
$$

We also consider the following hypothesis under which $(\mathcal{F}, \mathbb{P})$-martingales remain $(\mathcal{G}, \mathbb{P})$-martingales.

Hypothesis H3. For all $s \leq t, \alpha_{t}(s)=\alpha_{s}(s)$.

Under Hypothesis H3, we can write the processes $F$ and $S$ as

$$
F_{t}:=\mathbb{P}\left(\tau \leq t \mid \mathcal{F}_{t}\right)=\sum_{s=0}^{t} \alpha_{s}(s), \quad S_{t}=1-F_{t}=\sum_{s=t+1}^{\infty} \alpha_{s}(s) .
$$

We also assume that $F_{t}<1$, for all $t \in[0, T]$. Then regarding the discrete-time process as a simple process in continuous-time setting, the following key lemma in [5] holds in our setting. 
Lemma 2. For any $t \geq s$, let $\xi \in L\left(\mathcal{F}_{t}\right)$. Then,

$$
\mathbb{E}\left(\xi I_{\tau>t} \mid \mathcal{G}_{s}\right)=\frac{I_{\tau>s}}{S_{s}} \mathbb{E}\left(\xi S_{t} \mid \mathcal{F}_{s}\right) .
$$

Based on the above lemma, we can obtain the following proposition.

Proposition 3. Under Hypothesises H1 and H3, we have the following properties:

(1). $\mathcal{F}$ is immersed in $\mathcal{G}$, i.e. any $(\mathcal{F}, \mathbb{P})$-martingale is also a $(\mathcal{G}, \mathbb{P})$-martingale.

(2). Define $I_{t}:=I_{\tau=t}-I_{\tau \geq t} \frac{\alpha_{t}(t)}{S_{t-1}}$, then $\mathbb{E}\left[I_{t+1} \mid \mathcal{G}_{t}\right]=0$ and $\mathbb{E}\left[M_{t+1} I_{t+1} \mid \mathcal{G}_{t}\right]=0$.

Proof. (1): According to the martingale representation theorem in [2], all the $(\mathcal{F}, \mathbb{P})$-martingales can be represented in terms of $M_{t}$. Therefore we need only to prove $\mathbb{E}\left[M_{t+1} \mid \mathcal{G}_{t}\right]=0$, i.e. $\mathbb{E}\left[X_{t+1} \mid \mathcal{F}_{t}\right]=\mathbb{E}\left[X_{t+1} \mid \mathcal{G}_{t}\right]$, for all $t$, which is equivalent to that for all $t$, all $A_{t} \in \mathcal{G}_{t}, \mathbb{E}\left[X_{t+1} I_{A_{t}}\right]=$ $\mathbb{E}\left[\mathbb{E}\left[X_{t+1} \mid \mathcal{F}_{t}\right] I_{A_{t}}\right]$. Define

$$
\mathcal{M}_{t}:=\left\{A: \mathbb{E}\left[X_{t+1} I_{A}\right]=\mathbb{E}\left[\mathbb{E}\left[X_{t+1} \mid \mathcal{F}_{t}\right] I_{A}\right]\right\} .
$$

With $t$ fixed, it is obvious that the set

$$
\mathcal{A}_{t}:=\left\{\{\tau=s\} \cap A_{t},\{\tau=s\} \cup A_{t}: s \leq t, A_{t} \in \mathcal{F}_{t}\right\}
$$

is a $\pi$-system that contains $\Omega$ and $\mathcal{G}_{t}=\sigma\left(\mathcal{A}_{t}\right)$. Applying Monotone Class Theorem for functions, we reduce ourselves to proving that $\mathcal{A}_{t} \subset \mathcal{M}_{t}$. Since $\mathcal{F}_{t} \subset \mathcal{M}_{t}$, we need only to prove that for all $s \leq t$ and all $A_{t} \in \mathcal{F}_{t},\left(\{\tau=s\} \cap A_{t}\right) \in \mathcal{M}_{t}$. In fact,

$$
\begin{aligned}
& \mathbb{E}\left[X_{t+1} I_{\tau=s} I_{A_{t}}\right]=\mathbb{E}\left[\mathbb{E}\left(X_{t+1} \mid \mathcal{F}_{t}\right) I_{\tau=s} I_{A_{t}}\right] \\
& \Leftrightarrow \mathbb{E}\left[X_{t+1} I_{A_{t}} \mathbb{E}\left(I_{\tau=s} \mid \mathcal{F}_{t+1}\right)\right]=\mathbb{E}\left[\mathbb{E}\left(X_{t+1} \mid \mathcal{F}_{t}\right) I_{A_{t}} \mathbb{E}\left(I_{\tau=s} \mid \mathcal{F}_{t}\right)\right] \\
& \Leftrightarrow \mathbb{E}\left[X_{t+1} I_{A_{t}} \alpha_{s}(s)\right]=\mathbb{E}\left[X_{t+1} I_{A_{t}} \alpha_{s}(s)\right],
\end{aligned}
$$

where the last equality results from Hypothesis H3.

$(2)$ :

$$
\begin{aligned}
& \mathbb{E}\left[I_{t+1} \mid \mathcal{G}_{t}\right]=\mathbb{E}\left[I_{\tau=t+1}-I_{\tau \geq t+1} \frac{\alpha_{t+1}(t+1)}{S_{t}} \mid \mathcal{G}_{t}\right] \\
& =\mathbb{E}\left[I_{\tau \geq t+1}-I_{\tau>t+1} \mid \mathcal{G}_{t}\right]-I_{\tau>t} \mathbb{E}\left[\frac{\alpha_{t+1}(t+1)}{S_{t}} \mid \mathcal{G}_{t}\right] \\
& =I_{\tau>t}-\frac{I_{\tau>t}}{S_{t}} \mathbb{E}\left[S_{t+1} \mid \mathcal{F}_{t}\right]-I_{\tau>t} \mathbb{E}\left[\frac{\alpha_{t+1}(t+1)}{S_{t}} \mid \mathcal{F}_{t}\right] \\
& =0
\end{aligned}
$$

where the last equality results from that $S_{t}$ is $\mathcal{F}_{t}$-measurable and $S_{t}-S_{t+1}=\alpha_{t+1}(t+1)$.

$$
\begin{aligned}
& \mathbb{E}\left[M_{t+1} I_{t+1} \mid \mathcal{G}_{t}\right]=\mathbb{E}\left[X_{t+1}\left(I_{\tau=t+1}-I_{\tau \geq t+1} \frac{\alpha_{t+1}(t+1)}{S_{t}}\right) \mid \mathcal{G}_{t}\right] \\
& =\mathbb{E}\left[X_{t+1}\left(I_{\tau \geq t+1}-I_{\tau>t+1}\right) \mid \mathcal{G}_{t}\right]-I_{\tau>t} \mathbb{E}\left[\frac{X_{t+1} \alpha_{t+1}(t+1)}{S_{t}} \mid \mathcal{G}_{t}\right] \\
& =I_{\tau>t} \mathbb{E}\left[X_{t+1} \mid \mathcal{F}_{t}\right]-\frac{I_{\tau>t}}{S_{t}} \mathbb{E}\left[X_{t+1} S_{t+1} \mid \mathcal{F}_{t}\right]-I_{\tau>t} \mathbb{E}\left[\frac{X_{t+1} \alpha_{t+1}(t+1)}{S_{t}} \mid \mathcal{F}_{t}\right] \\
& =0 .
\end{aligned}
$$

Remark 4. Throughout this paper, we assume that Hypothesises H1, H2 and H3 hold. 


\section{Martingale representation theorem under progressively enlarged filtration}

Martingale representation theorem plays an important role in establishing existence and uniqueness theorem. Before giving the martingale representation theorem, we need the following definition.

Definition 5. For any $\mathcal{G}$-adapted processes $Z$ in $\mathbb{R}^{1 \times N}$ and $U$ in $\mathbb{R}$, define the seminorms of $Z$ and $U,\|Z\|_{M}$ and $\|U\|_{I}$, respectively by:

$$
\|Z\|_{M}^{2}:=\mathbb{E} \sum_{0 \leq s<T}\left(Z_{s} M_{s+1}\right)^{2}, \text { and }\|U\|_{I}^{2}:=\mathbb{E} \sum_{0 \leq s<T}\left(U_{s} I_{s+1}\right)^{2} .
$$

We shall write $Z^{1} \sim_{M} Z^{2}$ if $\left\|Z^{1}-Z^{2}\right\|_{M}^{2}=0$, and write $U^{1} \sim_{I} U^{2}$ if $\left\|U^{1}-U^{2}\right\|_{I}^{2}=0$. Also we shall write $Z_{t}^{1} \sim_{M_{t+1}} Z_{t}^{2}$ if $Z_{t}^{1} M_{t+1}=Z_{t}^{2} M_{t+1}$, and write $U_{t}^{1} \sim_{I_{t+1}} U_{t}^{2}$ if $U_{t}^{1} I_{t+1}=U_{t}^{2} I_{t+1}$.

Then we can obtain the following martingale representation theorem under progressively enlarged filtration.

Theorem 6. For any $\mathcal{G}$-adapted, $\mathbb{R}$-valued martingale $L$, there exists a pair of $\mathcal{G}$-adapted processes $Z \in \mathbb{R}^{1 \times N}$ and $U \in \mathbb{R}$ such that

$$
L_{t}=L_{0}+\sum_{0 \leq s<t} Z_{s} M_{s+1}+\sum_{0 \leq s<t} U_{s} I_{s+1} .
$$

These processes are unique up to equivalence $\sim_{M}$ and equivalence $\sim_{I}$ respectively.

Proof. Existence: As $L$ is $\mathcal{G}$-adapted, applying the Doob-Dynkin Lemma in [9], for each $t$, there exists some Borel-measurable function $h: \mathbb{R}^{N} \times \mathbb{R}^{N} \times \cdots \times \mathbb{R}^{N} \times \mathbb{R} \rightarrow \mathbb{R}$ such that

$$
L_{t+1}=L_{t}+h\left(X_{0}, X_{1}, \ldots, X_{t}, X_{t+1}, \tau \wedge(t+2)\right) .
$$

For simplicity, we take the terms $X_{0}, X_{1}, \ldots, X_{t-1}$ as implicit. We define $\tilde{Z}^{1}$ and $\tilde{Z}^{2}$ as

$$
\begin{aligned}
& \tilde{Z}_{t}^{1}:=\left[h\left(X_{t}, e_{1}, \tau \wedge(t+1)\right), h\left(X_{t}, e_{2}, \tau \wedge(t+1)\right), \ldots, h\left(X_{t}, e_{N}, \tau \wedge(t+1)\right)\right], \\
& \tilde{Z}_{t}^{2}:=\left[h\left(X_{t}, e_{1}, t+2\right), h\left(X_{t}, e_{2}, t+2\right), \ldots, h\left(X_{t}, e_{N}, t+2\right)\right] .
\end{aligned}
$$

According to Hypothesis H2, it is easy to check that

$$
L_{t+1}=L_{t}+\left(\tilde{Z}_{t}^{1} I_{\tau \leq t}+\tilde{Z}_{t}^{2} I_{\tau>t}\right) X_{t+1}+\left(h\left(X_{t}, X_{t}, t+1\right)-h\left(X_{t}, X_{t}, t+2\right)\right) I_{\tau=t+1} .
$$

Taking conditional expectation on both sides gives

$$
\left(\tilde{Z}_{t}^{1} I_{\tau \leq t}+\tilde{Z}_{t}^{2} I_{\tau>t}\right) \mathbb{E}\left[X_{t+1} \mid \mathcal{F}_{t}\right]+\left(h\left(X_{t}, X_{t}, t+1\right)-h\left(X_{t}, X_{t}, t+2\right)\right) \mathbb{E}\left[I_{\tau=t+1} \mid \mathcal{G}_{t}\right]=0 .
$$

It follows that

$L_{t+1}=L_{t}+\left(\tilde{Z}_{t}^{1} I_{\tau \leq t}+\tilde{Z}_{t}^{2} I_{\tau>t}\right) M_{t+1}+\left(h\left(X_{t}, X_{t}, t+1\right)-h\left(X_{t}, X_{t}, t+2\right)\right)\left(I_{\tau=t+1}-I_{\tau>t} \frac{\alpha_{t}(t+1)}{S_{t}}\right)$.

Since $\left\{\alpha_{s}(t+1)\right\}_{0 \leq s \leq T}$ is a $\mathcal{F}$-martingale, according to the martingale representation theorem in [2], there exists a process $\hat{Z}$ such that

$$
\alpha_{t+1}(t+1)=\alpha_{t}(t+1)+\hat{Z}_{t} M_{t+1} .
$$

Then we can obtain that

$$
L_{t+1}=L_{t}+Z_{t} M_{t+1}+U_{t} I_{t+1},
$$

where,

$$
\begin{aligned}
& Z_{t}=\left(\tilde{Z}_{t}^{1} I_{\tau \leq t}+\tilde{Z}_{t}^{2} I_{\tau>t}\right)+\left(h\left(X_{t}, X_{t}, t+1\right)-h\left(X_{t}, X_{t}, t+2\right)\right) I_{\tau>t} \frac{\hat{Z}_{t}}{S_{t}}, \\
& U_{t}=h\left(X_{t}, X_{t}, t+1\right)-h\left(X_{t}, X_{t}, t+2\right) .
\end{aligned}
$$

Uniqueness: If we had two possible solutions, $\left(Z^{1}, U^{1}\right)$ and $\left(Z^{2}, U^{2}\right)$, then it is obvious that

$$
\left[\left(Z_{t}^{1}-Z_{t}^{2}\right) M_{t+1}+\left(U_{t}^{1}-U_{t}^{2}\right) I_{t+1}\right]^{2}=0
$$

Taking expectation on both sides gives $Z_{t}^{1} M_{t+1}=Z_{t}^{2} M_{t+1}$ and $U_{t}^{1} I_{t+1}=U_{t}^{2} I_{t+1}$, for all $t$. Hence, $Z^{1} \sim_{M} Z^{2}$ and $U^{1} \sim_{I} U^{2}$. 


\section{Existence and uniqueness of BSDE solution}

In this paper, we considered BSDEs of the following form:

$$
Y_{t \wedge \tau}-\sum_{s=t \wedge \tau}^{T \wedge \tau-1} g\left(s, Y_{s}, Z_{s}, U_{s}\right)+\sum_{s=t \wedge \tau}^{T \wedge \tau-1} Z_{s} M_{s+1}+\sum_{s=t \wedge \tau}^{T \wedge \tau-1} U_{s} I_{s+1}=Q,
$$

under progressively enlarged $\sigma$-algebra $\left(\mathcal{G}_{t}\right)_{0 \leq t \leq T}$ and with terminal condition $Q \in L^{1}\left(\Omega, \mathcal{G}_{T \wedge \tau}, \mathbb{P}\right)$.

We will study the solutions to Equation (3) defined as follows:

Definition 7. Consider $T>0$ and $Q \in L^{1}\left(\Omega, \mathcal{G}_{T \wedge \tau}, \mathbb{P}\right)$. A solution to Equation (3) is a triple $\mathcal{G}$-adapted $\mathbb{R} \times \mathbb{R}^{1 \times N} \times \mathbb{R}$-valued process $(Y, Z, U)$ such that

1. $(Y, Z, U)$ is adapted and essentially bounded,

2. On the set of $\{t \geq T \wedge \tau\}$, we have $Y_{t}=Q, Z_{t}=0$ and $U_{t}=0$,

3. For all $t \in[0, T]$, we have

$$
Y_{t \wedge \tau}-\sum_{s=t \wedge \tau}^{T \wedge \tau-1} g\left(s, Y_{s}, Z_{s}, U_{s}\right)+\sum_{s=t \wedge \tau}^{T \wedge \tau-1} Z_{s} M_{s+1}+\sum_{s=t \wedge \tau}^{T \wedge \tau-1} U_{s} I_{s+1}=Y_{T \wedge \tau} .
$$

Remark 8. According to Definition 7, BSDE (3) is equivalent to the following one-step equation.

$$
\left\{\begin{array}{l}
I_{\tau>t} Y_{t+1}=I_{\tau>t}\left[Y_{t}-g\left(t, Y_{t}, Z_{t}, U_{t}\right)+Z_{t} M_{t+1}+U_{t} I_{t+1}\right], \forall t \in[0, T] \\
I_{\tau \leq t} Y_{t}=I_{\tau \leq t} Q, \forall t \in[0, T] \\
Y_{T}=Q
\end{array}\right.
$$

And from (4), properties of solution to BSDE (3) only depend on $I_{\tau>t} g(t, y, z, u)$, i.e. we don't need the whole information about $g$, but only the part in $\{\tau>t\}$.

Now, we establish the following theorem that guarantees the existence and uniqueness of solution to BSDE (3).

Theorem 9. Suppose $g$ satisfies the following assumptions:

(i). If $Z_{t}^{1} \sim_{M_{t+1}} Z_{t}^{2}$ and $U_{t}^{1} \sim_{I_{t+1}} U_{t}^{2}$, then for any $Y, g\left(\omega, t, Y_{t}, Z_{t}^{1}, U_{t}^{1}\right)=g\left(\omega, t, Y_{t}, Z_{t}^{2}, U_{t}^{2}\right)$ $\mathbb{P}$-a.s. for all t;

(ii). For any $(z, u) \in \mathbb{R}^{1 \times N} \times \mathbb{R}$, for all $t$, for $\mathbb{P}$-almost all $\omega$, the map

$$
y \mapsto y-g(\omega, t, y, z, u)
$$

is a bijection $\mathbb{R} \rightarrow \mathbb{R}$.

Then for any terminal condition $Q$ which is essentially bounded, $\mathcal{G}_{T \wedge \tau}$-measurable, and with values in $\mathbb{R}$, the BSDE (3) has an adapted solution $(Y, Z, U)$. Moreover, this solution is unique up to indistinguishability for $Y$, equivalence $\sim_{M}$ for $Z$ and equivalence $\sim_{I}$ for $U$.

Proof. Analogous with [2], we can find an (adapted) solution $Y_{T}=Q$ at time $T$, then construct the solution for all $t$ using backward induction.

Suppose we have a solution at time $t+1$. Taking a conditional expectation on both sides of the one-step equation (4) gives

$$
I_{\tau>t}\left[Y_{t}-g\left(t, Y_{t}, Z_{t}, U_{t}\right)\right]=I_{\tau>t} \mathbb{E}\left(Y_{t+1} \mid \mathcal{G}_{t}\right),
$$

and the martingale difference term must be

$$
I_{\tau>t}\left[Z_{t} M_{t+1}+U_{t} I_{t+1}\right]=I_{\tau>t}\left[Y_{t+1}-\mathbb{E}\left(Y_{t+1} \mid \mathcal{G}_{t}\right)\right] .
$$


According to Theorem 6 , there exists a unique $\tilde{Z}_{t}$ and $\tilde{U}$ up to equivalence $\sim_{M_{t+1}}$ and equivalence $\sim_{I_{t+1}}$ respectively such that

$$
\tilde{Z}_{t} M_{t+1}+\tilde{U}_{t} I_{t+1}=I_{\tau>t}\left[Z_{t} M_{t+1}+U_{t} I_{t+1}\right]=I_{\tau>t}\left[Y_{t+1}-\mathbb{E}\left(Y_{t+1} \mid \mathcal{G}_{t}\right)\right] .
$$

It follows that $I_{\tau>t} Z_{t}=\tilde{Z}_{t}$ and $I_{\tau>t} U_{t}=\tilde{U}_{t}$ up to equivalence $\sim_{M_{t+1}}$ and equivalence $\sim_{I_{t+1}}$ respectively. Since $I_{\tau \leq t} Z_{t}=0$ and $I_{\tau \leq t} U_{t}=0$, we have $Z_{t}=\tilde{Z}_{t}$ and $U_{t}=\tilde{U}_{t}$, which is unique up to equivalence $\sim_{M_{t+1}}$ and equivalence $\sim_{I_{t+1}}$ respectively.

Using such $Z_{t}$ and $U_{t}$, consider Equation (5). By Assumption (ii), for almost all $\omega \in\{\tau>t\}$, this equation has a unique solution $\tilde{Y}_{t}$. Since $I_{\tau \leq t} Y_{t}=Q$, we have $Y_{t}=\tilde{Y}_{t} I_{\tau>t}+Q I_{\tau \leq t}$. By such backward induction, we can obtain a unique solution $(Y, Z, U)$ up to indistinguishability for $Y$, equivalence $\sim_{M}$ for $Z$ and equivalence $\sim_{I}$ for $U$.

\section{Comparison theorem}

Comparison theorem is a key result in theory of BSDEs. It guarantees the monotonicity property of nonlinear expectations and risk measures defined as solutions of BSDEs. We now present a comparison theorem for discrete-time BSDEs with random terminal horizon. For ease of notation we make the following definition:

Definition 10. Let $\mathbb{I}_{t}$ denote the $\mathcal{G}_{t}$ measurable set of indices of possible values of $X_{t+1}$ and $I_{\tau=t+1}$, given $\mathcal{G}_{t}$, and $\mathbb{J}_{t}$ the $\mathcal{G}_{t}$-measurable set of indices of possible values of $X_{t+1}$ when $\tau \neq t+1$, given $\mathcal{G}_{t}$. That is,

$$
\begin{aligned}
\mathbb{I}_{t} & :=\left\{(i, j): \mathbb{P}\left(I_{\tau=t+1}=i, X_{t+1}=e_{j} \mid \mathcal{G}_{t}\right)>0\right\} \\
\mathbb{J}_{t} & :=\left\{j: \mathbb{P}\left(\tau \neq t+1, X_{t+1}=e_{j} \mid \mathcal{G}_{t}\right)>0\right\} \\
& =\left\{j: \mathbb{P}\left(X_{t+1}=e_{i} \mid \mathcal{F}_{t}\right)-\frac{I_{\tau>t}}{S_{t}} \mathbb{E}\left(\alpha_{t+1}(t+1) I_{X_{t+1}=e_{j}} \mid \mathcal{F}_{t}\right)>0\right\} .
\end{aligned}
$$

Applying the Doob-Dynkin Lemma in [9], for $\mathcal{F}_{t+1}$-measurable random variable $\alpha_{t+1}(t+1)$, there exists some Borel-measurable function $\alpha_{t+1}^{t+1}: \mathbb{R}^{N} \times \mathbb{R}^{N} \times \cdots \times \mathbb{R}^{N} \rightarrow \mathbb{R}$ such that

$$
\alpha_{t+1}(t+1)=\alpha_{t+1}^{t+1}\left(X_{0}, X_{1}, \ldots, X_{t}, X_{t+1}\right) .
$$

For simplicity, we take the terms $X_{0}, X_{1}, \ldots, X_{t}$ as implicit if no ambiguity. Then we define, for all $\mathcal{G}_{t}$-measurable random variables $Z_{t}^{k}$ and $U_{t}^{k}, k=1,2$,

$$
\begin{aligned}
\Phi_{t} & \left(Z_{t}^{1}, U_{t}^{1}, Z_{t}^{2}, U_{t}^{2}\right) \\
:= & \min _{(i, j) \in \mathbb{I}_{t}}\left\{\left(Z_{t}^{1}-Z_{t}^{2}\right)\left(e_{j}-\mathbb{E}\left(X_{t+1} \mid \mathcal{F}\right)\right)+\left(U_{t}^{1}-U_{t}^{2}\right)\left[i-\frac{\alpha_{t+1}^{t+1}\left(e_{j}\right)}{S_{t}}\right]\right\} \\
= & \min \left\{\min _{j \in \mathbb{J}_{t}}\left\{\left(Z_{t}^{1}-Z_{t}^{2}\right)\left(e_{j}-\mathbb{E}\left(X_{t+1} \mid \mathcal{F}\right)\right)-\left(U_{t}^{1}-U_{t}^{2}\right) \frac{\alpha_{t+1}^{t+1}\left(e_{j}\right)}{S_{t}}\right\},\right. \\
& \left.\left(Z_{t}^{1}-Z_{t}^{2}\right)\left(X_{t}-\mathbb{E}\left(X_{t+1} \mid \mathcal{F}\right)\right)+\left(U_{t}^{1}-U_{t}^{2}\right)\left[1-\frac{\alpha_{t+1}^{t+1}\left(X_{t}\right)}{S_{t}}\right]\right\},
\end{aligned}
$$

where the second equality results from Hypothesis H2, i.e. that if $\tau=t+1, X_{t+1}=X_{t}$.

Remark 11. In the case where $\tau$ is independent of $\mathcal{F}$, for example if we consider $\tau$ as death time of the insured, $\alpha_{s}(t)=p_{t}$ is a constant for all $s, t \in[0, T]$, where $p_{t}:=\mathbb{E}(\tau=t)$. Then $\Phi_{t}$ could have a simple form.

$$
\begin{aligned}
\Phi_{t} & \left(Z_{t}^{1}, U_{t}^{1}, Z_{t}^{2}, U_{t}^{2}\right) \\
= & \min \left\{\min _{j \in \mathbb{J}_{t}}\left\{\left(Z_{t}^{1}-Z_{t}^{2}\right)\left(e_{j}-\mathbb{E}\left(X_{t+1} \mid \mathcal{F}\right)\right)-\left(U_{t}^{1}-U_{t}^{2}\right) \frac{p_{t+1}}{S_{t}}\right\},\right. \\
& \left.\left(Z_{t}^{1}-Z_{t}^{2}\right)\left(X_{t}-\mathbb{E}\left(X_{t+1} \mid \mathcal{F}\right)\right)+\left(U_{t}^{1}-U_{t}^{2}\right)\left[1-\frac{p_{t+1}}{S_{t}}\right]\right\} .
\end{aligned}
$$


Theorem 12. Consider two BSDEs as in (3) with drivers $g^{i}, i=1,2$ and essentially bounded terminal values $Q^{i} \in L^{1}\left(\mathcal{G}_{T \wedge \tau}, \mathbb{P}\right)$. Suppose that $g^{i}$ satisfies both Assumptions (i) and (ii) in Theorem 9. Let $\left(Y^{i}, Z^{i}, U^{i}\right)$ be the associated solutions. Suppose the following conditions hold:

(i). $Q^{1} \geq Q^{2}, \mathbb{P}$-a.s.

(ii). $\mathbb{P}$-a.s., on $\{\tau>t\}, g^{1}\left(\omega, t, Y_{t}^{2}, Z_{t}^{2}, U_{t}^{2}\right) \geq g^{2}\left(\omega, t, Y_{t}^{2}, Z_{t}^{2}, U_{t}^{2}\right)$.

(iii). $\mathbb{P}$-a.s., on $\{\tau>t\}, g^{1}$ satisfies

$$
g^{1}\left(\omega, t, Y_{t}^{2}, Z_{t}^{1}, U_{t}^{1}\right)-g^{1}\left(\omega, t, Y_{t}^{2}, Z_{t}^{2}, U_{t}^{2}\right) \geq \Phi_{t}\left(Z_{t}^{1}, U_{t}^{1}, Z_{t}^{2}, U_{t}^{2}\right) .
$$

(iv). $\mathbb{P}$-a.s., on $\{\tau>t\}$, if

$$
Y_{t}^{1}-g^{1}\left(\omega, t, Y_{t}^{1}, Z_{t}^{1}, U_{t}^{1}\right) \geq Y_{t}^{2}-g^{1}\left(\omega, t, Y_{t}^{2}, Z_{t}^{1}, U_{t}^{1}\right),
$$

then $Y_{t}^{1} \geq Y_{t}^{2}$.

Then $Y^{1} \geq Y^{2} \mathbb{P}$-a.s.

Proof. We will prove this theorem using backward induction. Throughout this proof, we omit $\omega$ and $t$ arguments for $g^{i}$. For $t=T$, it is obvious that $Y_{T}^{1}=Q^{1} \geq Q^{2}=Y_{T}^{2}$. For $i=1,2$, taking the one-step equation (4), we have

$$
I_{\tau>t}\left[Y_{t}^{i}-g^{i}\left(t, Y_{t}^{i}, Z_{t}^{i}, U_{t}^{i}\right)+Z_{t}^{i} M_{t+1}+U_{t}^{i} I_{t+1}\right]=I_{\tau>t} Y_{t+1}^{i} .
$$

Suppose we have already obtained that $Y_{t+1}^{1} \geq Y_{t+1}^{2} \mathbb{P}$-a.s.. Then, on $\{\tau>t\}$,

$$
\begin{gathered}
Y_{t}^{1}-Y_{t}^{2}-g^{1}\left(Y_{t}^{1}, Z_{t}^{1}, U_{t}^{1}\right)+g^{2}\left(Y_{t}^{2}, Z_{t}^{2}, U_{t}^{2}\right)+\left(Z_{t}^{1}-Z_{t}^{2}\right) M_{t+1}+\left(U_{t}^{1}-U_{t}^{2}\right) I_{t+1} \\
=Y_{t+1}^{1}-Y_{t+1}^{2} \geq 0 .
\end{gathered}
$$

Note that $X_{t+1}$ takes values from the basis vector $e_{i}$ and that if $\tau=t+1, X_{t+1}=X_{t}$, therefore we can see

$$
Y_{t}^{1}-Y^{2} \geq g^{1}\left(Y_{t}^{1}, Z_{t}^{1}, U_{t}^{1}\right)-g^{2}\left(Y_{t}^{2}, Z_{t}^{2}, U_{t}^{2}\right)-\Phi_{t}\left(Z_{t}^{1}, U_{t}^{1}, Z_{t}^{2}, U_{t}^{2}\right) .
$$

Then Assumptions (ii) and (iii) imply

$$
\begin{aligned}
& Y_{t}^{1}-Y^{2}-g^{1}\left(Y_{t}^{1}, Z_{t}^{1}, U_{t}^{1}\right)+g^{1}\left(Y_{t}^{2}, Z_{t}^{1}, U_{t}^{1}\right) \\
& \geq {\left[g^{1}\left(Y_{t}^{2}, Z_{t}^{2}, U_{t}^{2}\right)-g^{2}\left(Y_{t}^{2}, Z_{t}^{2}, U_{t}^{2}\right)\right] } \\
& \quad+g^{1}\left(Y_{t}^{2}, Z_{t}^{1}, U_{t}^{1}\right)-g^{1}\left(Y_{t}^{2}, Z_{t}^{2}, U_{t}^{2}\right)-\Phi_{t}\left(Z_{t}^{1}, U_{t}^{1}, Z_{t}^{2}, U_{t}^{2}\right) .
\end{aligned}
$$

This implies

$$
Y_{t}^{1}-g^{1}\left(\omega, t, Y_{t}^{1}, Z_{t}^{1}, U_{t}^{1}\right) \geq Y_{t}^{2}-g^{1}\left(\omega, t, Y_{t}^{2}, Z_{t}^{1}, U_{t}^{1}\right)
$$

By Assumption (iv), it follows that $Y_{t}^{1} \geq Y_{t}^{2}$, P -a.s. on $\{\tau>t\}$. Since $Y_{t}^{1} I_{\tau \leq t}=Q^{1} I_{\tau \leq t} \geq$ $Q^{2} I_{\tau \leq t}=Y_{t}^{2} I_{\tau \leq t}$, we conclude that $Y_{t}^{1} \geq Y_{t}^{2} \mathbb{P}$-a.s.

Corollary 13. Suppose $g^{1}$ and $g^{2}$ satisfy the assumptions in Theorem 12 and the inequality in Assumption (iii) is strict, that is,

$$
g^{1}\left(\omega, t, Y_{t}^{2}, Z_{t}^{1}, U_{t}^{1}\right)-g^{1}\left(\omega, t, Y_{t}^{2}, Z_{t}^{2}, U_{t}^{2}\right)>\Phi_{t}\left(Z_{t}^{1}, U_{t}^{1}, Z_{t}^{2}, U_{t}^{2}\right),
$$

unless $Z_{t}^{1} \sim_{M_{t+1}} Z_{t}^{2}$ and $U_{t}^{1} \sim_{I_{t+1}} U_{t}^{2}$.

Then the comparison is strict in the sense that if on some $A \in \mathcal{G}_{t}, Y_{t}^{1}=Y_{t}^{2} \mathbb{P}$-a.s., then $\mathbb{P}$ a.s. on $A, Q^{1}=Q^{2}$ and for all $s \in\{t, \ldots, T\}, I_{\tau>s} g^{1}\left(s, Y_{s}^{2}, Z_{s}^{2}, U_{s}^{2}\right)=I_{\tau>s} g^{2}\left(s, Y_{s}^{2}, Z_{s}^{2}, U_{s}^{2}\right)$, $Z_{s}^{1} \sim_{M_{s+1}} Z_{s}^{2}, U_{s}^{1} \sim_{I_{s+1}} U_{s}^{2}$ and $Y_{s}^{1}=Y_{s}^{2}$. 
Proof. Throughout this proof, we omit $\omega$ and $t$ arguments for $g^{i}$. For a given $t$, by the same argument in the proof of Theorem 12, we can obtain, on $A \cap\{\tau>t\}$,

$$
\begin{aligned}
Y_{t}^{1}-Y^{2}-g^{1}\left(Y_{t}^{1}, Z_{t}^{1}, U_{t}^{1}\right)+g^{1}\left(Y_{t}^{2}, Z_{t}^{1}, U_{t}^{1}\right) \\
=\left[Y_{t+1}^{1}-Y_{t+1}^{2}\right]+\left[g^{1}\left(Y_{t}^{2}, Z_{t}^{2}, U_{t}^{2}\right)-g^{2}\left(Y_{t}^{2}, Z_{t}^{2}, U_{t}^{2}\right)\right] \\
\quad+g^{1}\left(Y_{t}^{2}, Z_{t}^{1}, U_{t}^{1}\right)-g^{1}\left(Y_{t}^{2}, Z_{t}^{2}, U_{t}^{2}\right)-\left(Z_{t}^{1}-Z_{t}^{2}\right) M_{t+1}-\left(U_{t}^{1}-U_{t}^{2}\right) I_{t+1} \\
\geq \\
\quad\left[g^{1}\left(Y_{t}^{2}, Z_{t}^{2}, U_{t}^{2}\right)-g^{2}\left(Y_{t}^{2}, Z_{t}^{2}, U_{t}^{2}\right)\right] \\
\quad+g^{1}\left(Y_{t}^{2}, Z_{t}^{1}, U_{t}^{1}\right)-g^{1}\left(Y_{t}^{2}, Z_{t}^{2}, U_{t}^{2}\right)-\Phi_{t}\left(Z_{t}^{1}, U_{t}^{1}, Z_{t}^{2}, U_{t}^{2}\right) \\
\geq 0,
\end{aligned}
$$

where the last inequality is strict unless $Z_{t}^{1} \sim_{M_{t+1}} Z_{t}^{2}$ and $U_{t}^{1} \sim_{I_{t+1}} U_{t}^{2}$ by assumption. Hence, if $Y_{t}^{1}=Y_{t}^{2}$, it is obvious that all the equalities holds, which is only the case if $Z_{t}^{1} \sim_{M_{t+1}} Z_{t}^{2}$ and $U_{t}^{1} \sim_{I_{t+1}} U_{t}^{2}$.

If $Z_{t}^{1} \sim_{M_{t+1}} Z_{t}^{2}$ and $U_{t}^{1} \sim_{I_{t+1}} U_{t}^{2}$, we have, $\mathbb{P}$-a.s.

$$
g^{1}\left(Y_{t}^{2}, Z_{t}^{1}, U_{t}^{1}\right)-g^{1}\left(Y_{t}^{2}, Z_{t}^{2}, U_{t}^{2}\right)-\left(Z_{t}^{1}-Z_{t}^{2}\right) M_{t+1}-\left(U_{t}^{1}-U_{t}^{2}\right) I_{t+1}=0 .
$$

Then we have

$$
0 \geq-\left[Y_{t+1}^{1}-Y_{t+1}^{2}\right]=g^{1}\left(Y_{t}^{2}, Z_{t}^{2}, U_{t}^{2}\right)-g^{2}\left(Y_{t}^{2}, Z_{t}^{2}, U_{t}^{2}\right) .
$$

Since the final term is nonnegative, all the terms are 0 , i.e.

$$
g^{1}\left(Y_{t}^{2}, Z_{t}^{2}, U_{t}^{2}\right)=g^{2}\left(Y_{t}^{2}, Z_{t}^{2}, U_{t}^{2}\right), \quad Y_{t+1}^{1}=Y_{t+1}^{2} .
$$

The argument on $\{\tau \leq t\}$ follows by Definition 7. Then the proof can be finished by forward induction.

\section{Applications}

In this section, we focus on applications of BSDEs in nonlinear expectations, as in [7] and [2-4], and optimal design of dynamic risk measures, as in [1] and [8].

\subsection{Nonlinear expectations}

The concept of nonlinear expectation was first introduced by Peng [7]. The classical nonlinear expectation was defined as a system of operators mapping from $L^{2}\left(\mathcal{F}_{T}\right)$ to $L^{2}\left(\mathcal{F}_{t}\right)$, where $\left(\mathcal{F}_{t}\right)_{0 \leq t \leq T}$ is the associated filtration. While, since we focus on the risk measures of defaultable contingent claims in this paper, a subtle modification is needed as follows.

Definition 14. A system of operators

$$
\mathcal{E}\left(\cdot \mid \mathcal{G}_{t}\right): L^{1}\left(\mathcal{G}_{T \wedge \tau}\right) \rightarrow L^{1}\left(\mathcal{G}_{t \wedge \tau}\right), \quad 0 \leq t \leq T
$$

is called a $\mathcal{G}_{t \wedge \tau}$-consistent nonlinear expectation if it satisfies the following properties:

(1). (Monotonicity) For any $Q \geq Q^{\prime} \mathbb{P}$-a.s.

$$
\mathcal{E}\left(Q \mid \mathcal{G}_{t \wedge \tau}\right) \geq \mathcal{E}\left(Q^{\prime} \mid \mathcal{G}_{t \wedge \tau}\right),
$$

$\mathbb{P}$-a.s. with equality only if $Q=Q^{\prime} \mathbb{P}$-a.s..

(2). ( $\mathcal{G}_{t \wedge \tau}$-triviality) $\mathcal{E}\left(Q \mid \mathcal{G}_{t \wedge \tau}\right)=Q \mathbb{P}$-a.s. for any $Q \in L^{2}\left(\mathcal{G}_{t \wedge \tau}, \mathbb{P}\right)$.

(3). (Recursivity) $\mathcal{E}\left(\mathcal{E}\left(Q \mid \mathcal{G}_{t \wedge \tau}\right) \mid \mathcal{G}_{s \wedge \tau}\right)=\mathcal{E}\left(Q \mid \mathcal{G}_{s \wedge \tau}\right) \mathbb{P}$-a.s. for any $s \leq t$.

(4). (Regularity) For any $A \in \mathcal{G}_{t \wedge \tau}, I_{A} \mathcal{E}\left(Q \mid \mathcal{G}_{t \wedge \tau}\right)=\mathcal{E}\left(I_{A} Q \mid \mathcal{G}_{t \wedge \tau}\right) \mathbb{P}$-a.s..

Definition 15. A $\mathcal{G}_{t \wedge \tau}$-consistent nonlinear expectation $\mathcal{E}\left(\cdot \mid \mathcal{G}_{t \wedge \tau}\right)$ is said to be (dynamically) translation invariant if for any $Q \in L^{1}\left(\mathcal{G}_{T \wedge \tau}\right)$, any $q \in L^{1}\left(\mathcal{G}_{t \wedge \tau}\right)$,

$$
\mathcal{E}\left(Q+q \mid \mathcal{G}_{t \wedge \tau}\right)=\mathcal{E}\left(Q \mid \mathcal{G}_{t \wedge \tau}\right)+q .
$$


We make the following definition that guarantees a comparison theorem, and further the monotonicity property of nonlinear expectations defined using BSDEs.

Definition 16. Consider a driver function $g$ satisfying Assumptions (i) and (ii) of Theorem 9. Suppose that, for each $t$, for any $Q^{1}, Q^{2} \in L^{1}\left(\mathcal{G}_{T \wedge \tau}\right)$, the corresponding BSDE solutions $\left(Y^{1}, Z^{1}, U^{1}\right)$, $\left(Y^{2}, Z^{2}, U^{2}\right)$ satisfy

(iii). P-a.s., on $\{\tau>t\}$,

$$
g\left(\omega, t, Y_{t}^{2}, Z_{t}^{1}, U_{t}^{1}\right)-g\left(\omega, t, Y_{t}^{2}, Z_{t}^{2}, U_{t}^{2}\right) \geq \Phi_{t}\left(Z_{t}^{1}, U_{t}^{1}, Z_{t}^{2}, U_{t}^{2}\right),
$$

with equality only if $Z_{t}^{1} \sim_{M_{t+1}} Z_{t}^{2}$ and $U_{t}^{1} \sim_{I_{t+1}} U_{t}^{2}$.

(iv). P-a.s., on $\{\tau>t\}$, if

$$
Y_{t}^{1}-g\left(\omega, t, Y_{t}^{1}, Z_{t}^{1}, U_{t}^{1}\right) \geq Y_{t}^{2}-g\left(\omega, t, Y_{t}^{2}, Z_{t}^{1}, U_{t}^{1}\right),
$$

then $Y_{t}^{1} \geq Y_{t}^{2}$.

(cf. Assumptions (iii) and (iv) of Theorem 12 and the assumption of Corollary 13). Then we shall call $g$ a balanced driver.

In the context of a discrete-time progressively enlarged filtration, we establish the following theorem which relates BSDEs to nonlinear expectations.

Theorem 17. For some family of operators $\mathcal{E}\left(\cdot \mid \mathcal{G}_{t \wedge \tau}\right): L^{1}\left(\mathcal{G}_{T \wedge \tau}\right) \rightarrow L^{1}\left(\mathcal{G}_{t \wedge \tau}\right)$, the following conditions are equivalent:

(i). $\mathcal{E}\left(\cdot \mid \mathcal{G}_{t \wedge \tau}\right)$ is a $\mathcal{G}_{t \wedge \tau}$-consistent, dynamically translation invariant nonlinear expectation.

(ii). There exists a driver $g$, which is balanced, independent of $y$ and satisfies $I_{\tau>t} g(t, y, 0,0)=0$, such that, for all $Q, Y_{t}=\mathcal{E}\left(Q \mid \mathcal{G}_{t \wedge \tau}\right)$ is the solution to a BSDE with terminal condition $Q$ and driver $g$.

Furthermore, these two statements are related by the equation

$$
I_{\tau>t} g(\omega, t, y, z, u)=\mathcal{E}\left(I_{\tau>t}\left(z M_{t+1}+u I_{t+1}\right) \mid \mathcal{G}_{t \wedge \tau}\right) .
$$

Proof. (ii) implies (i): Let $\mathcal{E}\left(Q \mid \mathcal{G}_{t \wedge \tau}\right):=Y_{t}$, the time $t$ solution of the BSDE with terminal value $Q$. We will prove that each property of a nonlinear expectation is satisfied.

(1). Monotonicity directly results from Theorem 12 and Corollary 13.

(2). By normalisation, the solution to the BSDE with $\mathcal{G}_{t \wedge \tau}$-measurable terminal condition $Q$ will be $\left(Y_{s}, Z_{s}, U_{s}\right)=(Q, 0,0)$ for $s \geq t$, i.e. $\mathcal{E}\left(Q \mid \mathcal{G}_{t \wedge \tau}\right)=Q$.

(3). Suppose the associated solution to the BSDE with driver $g$ and terminal condition $Q$ is $(Y, Z, U)$. Then it is obvious that $(\hat{Y}, \hat{Z}, \hat{U})$ defined as

$$
\left(\hat{Y}_{s}, \hat{Z}_{s}, \hat{U}_{s}\right)=\left\{\begin{array}{l}
\left(Y_{t}, 0,0\right), \quad t<s \leq T \\
\left(Y_{s}, Z_{s}, U_{s}\right), \quad 0 \leq s \leq t .
\end{array}\right.
$$

is the solution to the BSDE with deriver $g$ and terminal condition $\mathcal{E}^{g}\left(Q \mid \mathcal{G}_{t \wedge \tau}\right)$, which implies $\mathcal{E}^{g}\left(\mathcal{E}^{g}\left(Q \mid \mathcal{G}_{t \wedge \tau}\right) \mid \mathcal{G}_{s \wedge \tau}\right)=\mathcal{E}^{g}\left(Q \mid \mathcal{G}_{s \wedge \tau}\right)$.

(4). By normalisation, multiplying by $I_{A}$ in Equation (3) gives

$$
\begin{aligned}
I_{A} Y_{t \wedge \tau}= & I_{A} Q+\sum_{s=t \wedge \tau}^{T \wedge \tau-1} g\left(s, I_{A} Z_{s}, I_{A} U_{s}\right) \\
& -\sum_{s=t \wedge \tau}^{T \wedge \tau-1} I_{A} Z_{s} M_{s+1}-\sum_{s=t \wedge \tau}^{T \wedge \tau-1} I_{A} U_{s} I_{s+1},
\end{aligned}
$$

which implies that $\left(I_{A} Y, I_{A} Z, I_{A} U\right)$ is the solution to the BSDE with driver $g$ and terminal condition $I_{A} Q$, as desired. 
Now we show that this nonlinear expectation is dynamically translation invariant. Simply adding $q$ on both sides of BSDE (3) gives $\tilde{Y}_{t}=Y_{t}+q$ is the solution to BSDE with terminal condition $Q+q$.

Finally for any $u \in \mathbb{R}, z \in \mathbb{R}^{1 \times N}, Y_{t} \in L^{1}\left(\mathcal{G}_{t \wedge \tau}\right)$ and $Q \in L^{1}\left(\mathcal{G}_{T \wedge \tau}\right)$, we define

$$
Y_{t+1}:=I_{\tau>t}\left(Y_{t}-g(t, z, u)+z M_{t+1}+u I_{t+1}\right)
$$

Taking a $\mathcal{G}_{t}$-conditional expectation gives

$$
\begin{aligned}
I_{\tau>t} g(t, z, u) & =Y_{t}-\mathbb{E}\left(Y_{t+1} \mid \mathcal{G}_{t}\right) \\
& =\mathcal{E}\left(Y_{t+1} \mid \mathcal{G}_{t \wedge \tau}\right)-\mathbb{E}\left(Y_{t+1} \mid \mathcal{G}_{t}\right) \\
& =\mathcal{E}\left(Y_{t+1}-\mathbb{E}\left(Y_{t+1} \mid \mathcal{G}_{t}\right) \mid \mathcal{G}_{t \wedge \tau}\right) \\
& =\mathcal{E}\left(I_{\tau>t}\left(z M_{t+1}+u I_{t+1}\right) \mid \mathcal{G}_{t \wedge \tau}\right),
\end{aligned}
$$

as desired.

(i) implies (ii): Define $Y_{t}:=\mathcal{E}\left(Q \mid \mathcal{G}_{t \wedge \tau}\right)$ and driver $g$ such that

$$
I_{\tau>t} g(t, y, z)=\mathcal{E}\left(I_{\tau>t}\left(z M_{t+1}+u I_{t+1}\right) \mid \mathcal{G}_{t \wedge \tau}\right) .
$$

Since $\mathcal{G}_{t \wedge \tau}$ is finite-dimensional, by similar argument as in the proof of Theorem 7 in [2], we can obtain, for any $\mathcal{G}_{t}$-measurable random variables $Z_{t}$ and $U_{t}$,

$$
\begin{gathered}
I_{\tau>t} g\left(\omega, t, y, Z_{t}(\omega)\right)=\mathcal{E}\left(I_{\tau>t}\left(Z_{t} M_{t+1}+U_{t} I_{t+1}\right) \mid \mathcal{G}_{t \wedge \tau}\right)(\omega), \\
I_{\tau>t} Y_{t+1}=I_{\tau>t}\left[Y_{t}-g\left(t, Y_{t}, Z_{t}, U_{t}\right)+Z_{t} M_{t+1}+U_{t} I_{t+1}\right] .
\end{gathered}
$$

It is obvious that $I_{\tau \leq t} Y_{t}=\mathcal{E}\left(I_{\tau \leq t} Q \mid \mathcal{G}_{t \wedge \tau}\right)=I_{\tau \leq t} Q$. According to Remark $8, \mathcal{E}\left(Q \mid \mathcal{G}_{t \wedge \tau}\right)$ satisfies the BSDE with driver $g$.

Now, we need only to show that the driver $g$ is balanced. Since $g$ is independent of $y$, it remains to prove $g$ satisfies the assumption in Corollary 13. Let $\left(Y^{i}, Z^{i}, U^{i}\right), i=i, 2$, the associated solution to BSDE with driver $g$ and terminal condition $Q^{i}$. It is obvious that

$$
I_{\tau>t}\left[\left(Z_{t}^{1} M_{t+1}+U_{t}^{1} I_{t+1}\right)-\Phi_{t}\left(Z_{t}^{1}, U_{t}^{1}, Z_{t}^{2}, U_{t}^{2}\right)\right] \geq I_{\tau>t}\left(Z_{t}^{2} M_{t+1}+U_{t}^{2} I_{t+1}\right) .
$$

By monotonicity of the nonlinear expectation,

$$
\begin{aligned}
& \mathcal{E}\left[I_{\tau>t}\left(\left(Z_{t}^{1} M_{t+1}+U_{t}^{1} I_{t+1}\right)-\Phi_{t}\left(Z_{t}^{1}, U_{t}^{1}, Z_{t}^{2}, U_{t}^{2}\right)\right) \mid \mathcal{G}_{t \wedge \tau}\right] \\
& \quad \geq \mathcal{E}\left[I_{\tau>t}\left(Z_{t}^{2} M_{t+1}+U_{t}^{2} I_{t+1}\right) \mid \mathcal{G}_{t \wedge \tau}\right],
\end{aligned}
$$

with equality only if the equality holds in Equation (7). By dynamic translation invariance of $\mathcal{E}$, it follows that

$$
\begin{aligned}
& \mathcal{E}\left[I_{\tau>t}\left(Z_{t}^{1} M_{t+1}+U_{t}^{1} I_{t+1}\right) \mid \mathcal{G}_{t \wedge \tau}\right]-\mathcal{E}\left[I_{\tau>t}\left(Z_{t}^{2} M_{t+1}+U_{t}^{2} I_{t+1}\right) \mid \mathcal{G}_{t \wedge \tau}\right] \\
& \geq I_{\tau>t} \Phi_{t}\left(Z_{t}^{1}, U_{t}^{1}, Z_{t}^{2}, U_{t}^{2}\right) .
\end{aligned}
$$

with equality only if equality holds in Equation (7). If equality holds in Equation (7) on $A \in \mathcal{G}_{t}$. Taking $\mathcal{G}_{t}$ conditional expectation on both sides of Equation (7) on $A$ gives

$$
\left(Z_{t}^{1}-Z_{t}^{2}\right) M_{t+1}+\left(U_{t}^{1}+U_{t}^{2}\right) I_{t+1}=0
$$

$\mathbb{P}$-a.s. on $A$. Hence $Z_{t}^{1} \sim_{M_{t+1}} Z_{t}^{2}$ and $U_{t}^{1} \sim_{I_{t+1}} U_{t}^{2}$ as required.

\subsection{Optimal design of dynamic risk measures}

The problem of default risk which is characterized by a single jump process was explored by Shen and Elliott [8]. However, in that work, the payment at default time is a constant. In this subsection, we will generalize this problem by considering $\mathcal{F}$-adapted terminal payment at expiration time $T$ and $\mathcal{F}$-predictable payment at default time. 
Definition 18. For any defaultable contingent claim with terminal payoff $Q \in L^{1}\left(\mathcal{G}_{T \wedge \tau}\right)$, the static and dynamic entropic risk measure (with tolerance coefficient $\gamma$ ) of this claim at time $t$ are defined as

$$
e^{\gamma}(Q):=\gamma \ln \mathbb{E} \exp \left(-\frac{Q}{\gamma}\right) \text { and } e_{t}^{\gamma}(Q):=\gamma \ln \mathbb{E}\left(\exp \left(-\frac{Q}{\gamma}\right) \mid \mathcal{G}_{t}\right) .
$$

We consider a problem about an optimal transaction between two economic agents, denoted by $A$ and $B$ respectively. Agent $A$ is exposed towards a non-hedgeable risk of a financial position $Q$ with terminal payment

$$
Q:=V I_{\tau>T}+C_{\tau} I_{\tau \leq T}
$$

where $V$ is $\mathcal{F}_{T}$-measurable, i.e. $V$ depends on the paths of asset prices until time $T$, and $C$ is an $\mathcal{F}$-predictable nonnegative process.

Thus, agent $A$ wants to issue a financial product $S$ and sell it to agent $B$ for a forward price at time $T$ denoted by $\pi$ to reduce his exposure.

Suppose both agents use entropic risk measures, with tolerance coefficients $\gamma$ and $\gamma^{\prime}$, to assess the risk of their financial positions. Agent $A$ wants to determine the structure $(S, \pi)$ as to minimize his global risk measure

$$
\inf _{S, \pi} e^{\gamma}(Q-S+\pi)
$$

with the constraint

$$
e^{\gamma^{\prime}}(S-\pi) \leq e^{\gamma^{\prime}}(0)=0 .
$$

Using the cash translation invariance property and binding the constraint at the optimum, the pricing rule of the $S$-structure is fully determined by the buyer as

$$
\pi^{*}(S)=-e^{\gamma^{\prime}}(S)
$$

Using the cash translation invariance property again, the optimization problem simply becomes

$$
\inf _{S}\left(e^{\gamma}(Q-S)+e^{\gamma^{\prime}}(S)\right)
$$

We have the following proposition.

Proposition 19. $-e_{t}^{\gamma}(Q)$ is an $\mathcal{G}_{t \wedge \tau}$-consistent, dynamically translation invariant nonlinear expectation.

Proof. Monotonicity, $\mathcal{G}_{t \wedge \tau}$-triviality, recursivity and dynamically translation invariance are obvious. We only show the proof for regularity. For any $A \in \mathcal{G}_{t \wedge \tau}$,

$$
\begin{aligned}
& -e_{t}^{\gamma}\left(I_{A} Q\right)=I_{A}\left(-e_{t}^{\gamma}(Q)\right) \\
& \Leftrightarrow \ln \mathbb{E}\left(\exp \left(-\frac{Q I_{A}}{\gamma}\right) \mid \mathcal{G}_{t}\right)=I_{A} \ln \mathbb{E}\left(\exp \left(-\frac{Q}{\gamma}\right) \mid \mathcal{G}_{t}\right) \\
& \Leftrightarrow \mathbb{E}\left(\exp \left(-\frac{Q I_{A}}{\gamma}\right) \mid \mathcal{G}_{t}\right)=\left[\mathbb{E}\left(\exp \left(-\frac{Q}{\gamma}\right) \mid \mathcal{G}_{t}\right)\right]^{I_{A}}
\end{aligned}
$$

It is obvious that

$$
\mathbb{E}\left(\exp \left(-\frac{Q I_{A}}{\gamma}\right) \mid \mathcal{G}_{t}\right) I_{A}=\left[\mathbb{E}\left(\exp \left(-\frac{Q}{\gamma}\right) \mid \mathcal{G}_{t}\right)\right]^{I_{A}} I_{A}=\mathbb{E}\left(\exp \left(-\frac{Q}{\gamma}\right) \mid \mathcal{G}_{t}\right) I_{A}
$$

and that

$$
\mathbb{E}\left(\exp \left(-\frac{Q I_{A}}{\gamma}\right) \mid \mathcal{G}_{t}\right) I_{A^{c}}=\left[\mathbb{E}\left(\exp \left(-\frac{Q}{\gamma}\right) \mid \mathcal{G}_{t}\right)\right]^{I_{A}} I_{A^{c}}=I_{A^{c}}
$$

Then combining the above proposition and Theorem 17, We can obtain the following theorem, which relates $e_{t}^{\gamma}(\cdot)$ to BSDEs under progressively enlarged filtration. 
Theorem 20. $\left(e_{t}^{\gamma}(Q-S), Z_{t}^{\gamma}, U^{\gamma}\right)$ is the solution of the following BSDE:

$$
e_{t}^{\gamma}(Q-S)-\sum_{s=t \wedge \tau}^{T \wedge \tau-1} g^{\gamma}\left(s, Y_{s}, Z_{s}^{\gamma}, U_{s}^{\gamma}\right)+\sum_{s=t \wedge \tau}^{T \wedge \tau-1} Z_{s}^{\gamma} M_{s+1}+\sum_{s=t \wedge \tau}^{T \wedge \tau-1} U_{s}^{\gamma} I_{s+1}=-(Q-S),
$$

where

$$
I_{\tau>t} g^{\gamma}(\omega, t, z, u)=\gamma \ln \mathbb{E}\left(\exp \left(\frac{I_{\tau>t}\left(z M_{t+1}+u I_{t+1}\right)}{\gamma}\right) \mid \mathcal{G}_{t}\right) .
$$

We now study for any $t \in[0, T]$ the inf-convolution of the dynamic entropic risk measures $e_{t}^{\gamma}$ and $e_{t}^{\gamma^{\prime}}$ and the inf-convolution of the corresponding $g_{t}^{\gamma}$ and $g_{t}^{\gamma}$. We define

$$
\begin{gathered}
\left(e^{\gamma} \square e^{\gamma^{\prime}}\right)_{t}(Q):=\inf _{S}\left(e_{t}^{\gamma}(Q-S)+e_{t}^{\gamma^{\prime}}(S)\right) \\
g^{\gamma} \square g^{\gamma^{\prime}}(\omega, t, z, u):=\inf _{z^{\prime}, u^{\prime}}\left(g^{\gamma}\left(\omega, t, z-z^{\prime}, u-u^{\prime}\right)+g^{\gamma^{\prime}}\left(\omega, t, z^{\prime}, u^{\prime}\right)\right)
\end{gathered}
$$

We finish this subsection by showing the following theorem, which characterizes $\left(e^{\gamma} \square e^{\gamma^{\prime}}\right)_{t}(Q)$ and $g^{\gamma} \square g^{\gamma^{\prime}}(\omega, t, z, u)$ and gives the optimal solution.

Theorem 21. Inf-convolutions (9) and (10) have properties as follows:

$$
\begin{aligned}
& I_{\tau>t} g^{\gamma} \square g^{\gamma^{\prime}}(\omega, t, z, u)=I_{\tau>t} g^{\gamma+\gamma^{\prime}}(\omega, t, z, u), \\
&\left(e^{\gamma} \square e^{\gamma^{\prime}}\right)_{t}(Q)= \sum_{s=t \wedge \tau}^{T \wedge \tau-1} g^{\gamma+\gamma^{\prime}}\left(s, Y_{s}, Z_{s}, U_{s}\right)-\sum_{s=t \wedge \tau}^{T \wedge \tau-1} Z_{s} M_{s+1} \\
&-\sum_{s=t \wedge \tau}^{T \wedge \tau-1} U_{s} I_{s+1}-Q=e_{t}^{\gamma+\gamma^{\prime}}(Q) .
\end{aligned}
$$

The solution to the dynamic optimal design problem is

$$
S^{*}=\frac{\gamma^{\prime}}{\gamma+\gamma^{\prime}} Q+c
$$

where $c$ is an arbitrary constant.

Proof. Consider the function

$$
\begin{aligned}
f\left(z^{\prime}, u^{\prime}\right):= & I_{\tau>t} g^{\gamma}\left(\omega, t, z-z^{\prime}, u-u^{\prime}\right)+I_{\tau>t} g^{\gamma^{\prime}}\left(\omega, t, z^{\prime}, u^{\prime}\right) \\
= & \gamma \ln \mathbb{E}\left(\exp \left(I_{\tau>t} \frac{\left(z-z^{\prime}\right) M_{t+1}+\left(u-u^{\prime}\right) I_{t+1}}{\gamma}\right) \mid \mathcal{G}_{t}\right) \\
& +\gamma^{\prime} \ln \mathbb{E}\left(\exp \left(I_{\tau>t} \frac{z^{\prime} M_{t+1}+u^{\prime} I_{t+1}}{\gamma^{\prime}}\right) \mid \mathcal{G}_{t}\right) .
\end{aligned}
$$

It is obvious that

$$
f^{*}\left(z^{\prime}, u^{\prime}\right):=\ln \mathbb{E}\left(\exp \left(I_{\tau>t} \frac{z^{\prime} M_{t+1}+u^{\prime} I_{t+1}}{\gamma^{\prime}}\right) \mid \mathcal{G}_{t}\right)
$$

is convex. It follows that $f\left(z^{\prime}, u^{\prime}\right)$ is convex. Therefore for each $\omega$ the minimum of (10) with respect to $\left(z^{\prime}, u^{\prime}\right)$ occurs when

$$
\begin{aligned}
\nabla f\left(z^{\prime}, u^{\prime}\right)=\frac{\mathbb{E}\left(I_{\tau>t}\left(M_{t+1}^{*}, I_{t+1}\right)\right.}{\left.\exp \left(\frac{z^{\prime} M_{t+1}+u^{\prime} I_{t+1}}{\gamma^{\prime}}\right) \mid \mathcal{G}_{t}\right)} \\
\mathbb{E}\left(\exp \left(I_{\tau>t} \frac{z^{\prime} M_{t+1}+u^{\prime} I_{t+1}}{\gamma^{\prime}}\right) \mid \mathcal{G}_{t}\right) \\
-\frac{\mathbb{E}\left(I_{\tau>t}\left(M_{t+1}^{*}, I_{t+1}\right) \exp \left(\frac{\left(z-z^{\prime}\right) M_{t+1}+\left(u-u^{\prime}\right) I_{t+1}}{\gamma}\right) \mid \mathcal{G}_{t}\right)}{\mathbb{E}\left(\exp \left(I_{\tau>t} \frac{\left(z-z^{\prime}\right) M_{t+1}+\left(u-u^{\prime}\right) I_{t+1}}{\gamma}\right) \mid \mathcal{G}_{t}\right)}=0 .
\end{aligned}
$$


Denote by $\left(z^{* \gamma^{\prime}}, u^{* \gamma^{\prime}}\right)$ the value at which the minimum is attained. It is obvious that $\left(z^{* \gamma^{\prime}}, u^{* \gamma^{\prime}}\right)$ is unique up to equivalence $\sim_{M_{t+1}}$ and $\sim_{I_{t+1}}$, and

Therefore

$$
z^{* \gamma^{\prime}}=\frac{\gamma^{\prime}}{\gamma+\gamma^{\prime}} z, \quad u^{* \gamma^{\prime}}=\frac{\gamma^{\prime}}{\gamma+\gamma^{\prime}} u
$$

$$
\begin{aligned}
I_{\tau>t} g^{\gamma} \square g^{\gamma^{\prime}}(\omega, s, z) & =\inf _{z^{\prime}}\left(I_{\tau>t} g^{\gamma}\left(\omega, t, z-z^{\prime}, u-u^{\prime}\right)+I_{\tau>t} g^{\gamma^{\prime}}\left(\omega, t, z^{\prime}, u^{\prime}\right)\right) \\
& =I_{\tau>t} g^{\gamma}\left(\omega, t, z-z^{* \gamma^{\prime}}, u-u^{* \gamma^{\prime}}\right)+I_{\tau>t} g^{\gamma^{\prime}}\left(\omega, t, z^{* \gamma^{\prime}}, u^{* \gamma^{\prime}}\right) \\
& =\left(\gamma+\gamma^{\prime}\right) \ln \mathbb{E}\left(\exp \left(I_{\tau>t} \frac{z M_{t+1}+u I_{t+1}}{\gamma+\gamma^{\prime}}\right) \mid \mathcal{G}_{t}\right) \\
& =I_{\tau>t} g^{\gamma+\gamma^{\prime}}(\omega, s, z, u) .
\end{aligned}
$$

Similar to (8), we have

$$
e_{t}^{\gamma^{\prime}}(S)-\sum_{s=t \wedge \tau}^{T \wedge \tau-1} g^{\gamma^{\prime}}\left(s, Z_{s}^{\gamma^{\prime}}, U_{s}^{\gamma^{\prime}}\right)+\sum_{s=t \wedge \tau}^{T \wedge \tau-1} Z_{s}^{\gamma^{\prime}} M_{s+1}+\sum_{s=t \wedge \tau}^{T \wedge \tau-1} U_{s}^{\gamma^{\prime}} I_{s+1}=-S .
$$

Adding (8) and (13), we have

$$
\begin{aligned}
& e_{t}^{\gamma}(Q-S)+e_{t}^{\gamma^{\prime}}(S) \\
& =\sum_{s=t \wedge \tau}^{T \wedge \tau-1}\left[g^{\gamma}\left(s, Z_{s}^{\gamma}, U_{s}^{\gamma}\right)+g^{\gamma^{\prime}}\left(s, Z_{s}^{\gamma^{\prime}}, U_{s}^{\gamma^{\prime}}\right)\right]-\sum_{s=t \wedge \tau}^{T \wedge \tau-1} Z_{s} M_{s+1}-\sum_{s=t \wedge \tau}^{T \wedge \tau-1} U_{s} I_{s+1}-Q \\
& =\sum_{s=t \wedge \tau}^{T \wedge \tau-1}\left[g^{\gamma}\left(s, Z_{s}-Z_{s}^{\gamma^{\prime}}, U_{s}-U_{s}^{\gamma^{\prime}}\right)+g^{\gamma^{\prime}}\left(s, Z_{s}^{\gamma^{\prime}}, U_{s}^{\gamma}\right)\right]-\sum_{s=t \wedge \tau}^{T \wedge \tau-1} Z_{s} M_{s+1}-\sum_{s=t \wedge \tau}^{T \wedge \tau-1} U_{s} I_{s+1}-Q \\
& \geq \sum_{s=t \wedge \tau}^{T \wedge \tau-1} g^{\gamma+\gamma^{\prime}}\left(s, Z_{s}, U_{s}\right)-\sum_{s=t \wedge \tau}^{T \wedge \tau-1} Z_{s} M_{s+1}-\sum_{s=t \wedge \tau}^{T \wedge \tau-1} U_{s} I_{s+1}-Q .
\end{aligned}
$$

where $Z_{s}=Z_{s}^{\gamma}+Z_{s}^{\gamma^{\prime}}$ and $U_{s}=U_{s}^{\gamma}+U_{s}^{\gamma^{\prime}}$. Thus, $e_{t}^{\gamma}(Q-S)+e_{t}^{\gamma^{\prime}}(S)$ can be regarded as the solution to the BSDE with terminal condition $-Q$ and the driver

$$
g(s, z, u)=g^{\gamma}\left(s, z-Z_{s}^{\gamma^{\prime}}, u-U_{s}^{\gamma^{\prime}}\right)+g^{\gamma^{\prime}}\left(s, Z_{s}^{\gamma^{\prime}}, U_{s}^{\gamma^{\prime}}\right) .
$$

Similar to $(8), e_{t}^{\gamma+\gamma^{\prime}}(Q)$ is the solution to the BSDE with terminal condition $-Q$ and the driver $g^{\gamma+\gamma^{\prime}} \cdot g \geq g^{\gamma+\gamma^{\prime}}$ implies that for any $S$,

$$
e_{t}^{\gamma}(Q-S)+e_{t}^{\gamma^{\prime}}(S) \geq e_{t}^{\gamma+\gamma^{\prime}}(Q) .
$$

Taking $S^{*}=\frac{\gamma^{\prime}}{\gamma+\gamma^{\prime}} Q$, we can show that $\gamma Z_{s}^{* \gamma^{\prime}} M_{s+1}=\gamma^{\prime} Z_{s}^{* \gamma} M_{s+1}$ and $\gamma U_{s}^{* \gamma^{\prime}} I_{s+1}=\gamma^{\prime} U_{s}^{* \gamma} I_{s+1}$. Then

$$
\begin{aligned}
& Z_{s}^{* \gamma^{\prime}} M_{s+1}=\frac{\gamma^{\prime}}{\gamma+\gamma^{\prime}}\left(Z_{s}^{* \gamma^{\prime}}+Z_{s}^{* \gamma}\right) M_{s+1}=\frac{\gamma^{\prime}}{\gamma+\gamma^{\prime}} Z_{s} M_{s+1} . \\
& U_{s}^{* \gamma^{\prime}} I_{s+1}=\frac{\gamma^{\prime}}{\gamma+\gamma^{\prime}}\left(U_{s}^{* \gamma^{\prime}}+U_{s}^{* \gamma}\right) I_{s+1}=\frac{\gamma^{\prime}}{\gamma+\gamma^{\prime}} U_{s} I_{s+1} .
\end{aligned}
$$

Consequently,

$$
e_{t}^{\gamma}\left(Q-S^{*}\right)+e_{t}^{\gamma^{\prime}}\left(S^{*}\right)=\sum_{s=t \wedge \tau}^{T \wedge \tau-1} g^{\gamma+\gamma^{\prime}}\left(s, Z_{s}, U_{s}\right)-\sum_{s=t \wedge \tau}^{T \wedge \tau-1} Z_{s} M_{s+1}-\sum_{s=t \wedge \tau}^{T \wedge \tau-1} U_{s} I_{s+1}-Q .
$$

This means $e_{t}^{\gamma}\left(Q-S^{*}\right)+e_{t}^{\gamma^{\prime}}\left(S^{*}\right)$ is the solution to the BSDE with the driver function $g^{\gamma+\gamma^{\prime}}$ and the terminal condition $-Q$. Then it also equals to $e_{t}^{\gamma+\gamma^{\prime}}(Q)$ due to the uniqueness of the solution. According to (14), we have

$$
e_{t}^{\gamma+\gamma^{\prime}}(Q)=\inf _{S}\left(e_{t}^{\gamma}(Q-S)+e_{t}^{\gamma^{\prime}}(S)\right)=\left(e^{\gamma} \square e^{\gamma^{\prime}}\right)_{t}(Q) .
$$




\section{Conclusion}

We have constructed discrete-time finite-state BSDEs under progressively enlarged filtration and established martingale representation theorem, existence and uniqueness theorem and a comparison theorem. Relationship between nonlinear expectations and solutions of BSDEs has been given under some mild assumptions. We have obtained the solution for optimal design of dynamic entropic risk measures of a defaultable contingent claim, which generalizes the work of Shen and Elliott [8].

Acknowledgements. We would like to thank the anonymous referee for the valuable comments and suggestions. We acknowledge with thanks the support from the Research Grants Council of the Hong Kong Special Administrative Region (project No. HKU 706611P).

\section{References}

[1] Barrieu, P., El Karoui, N., 2004. Optimal derivatives design under dynamic risk measures. Contemporary Mathematics 351, 13-26.

[2] Cohen, S. N., Elliott, R. J., 2010a. A general theory of finite state backward stochastic difference equations. Stochastic Processes and their Applications 120 (4), 442-466.

[3] Cohen, S. N., Elliott, R. J., 2010b. Comparisons for backward stochastic differential equations on markov chains and related no-arbitrage conditions. The Annals of Applied Probability 20 (1), $267-311$.

[4] Cohen, S. N., Elliott, R. J., 2012. Existence, uniqueness and comparisons for bsdes in general spaces. The Annals of Probabilty 40 (5), 2264-2297.

[5] Dellacherie, C., 1970. Un exemple de la théorie générale des processus. In: Meyer, P. A. (Ed.), Séminaire de Probabilités IV. Springer, Berlin Heidelberg New York, pp. 60-70.

[6] Jeanblanc, M., Le Cam, Y., 2009. Progressive enlargement of filtrations with initial times. Stochastic Processes and their Applications 119 (8), 2523-2543.

[7] Peng, S., 2004. Nonlinear expectations, nonlinear evaluations and risk measures. In: Stochastic Methods in Finance. Springer, Berlin Heidelberg, pp. 165-253.

[8] Shen, L., Elliott, R., 2012. Optimal design of dynamic default risk measures. J. Appl. Probab. 49 (4), 967-977.

[9] Shiryaev, A. N., 2000. Probability, 2nd Edition. Springer. 